\title{
Treatment outcome of hepatocellular carcinoma patients with high-risk vascular invasion: a retrospective analysis
}

\author{
Jade Suphapol, Boonchoo Sirichindakul, Bunthoon Nonthasoot, Supanit Nivatvongs \\ Department of Surgery, Faculty of Medicine, Chulalongkorn University, Bangkok 10330, Thailand
}

\begin{abstract}
Background: Invasion of major hepatic vessels in hepatocellular carcinoma (HCC) generally prohibits the surgical treatment.

Objective: Analyze outcomes of non-surgical approaches in this group of HCC.

Methods: Retrospective review of medical records of 648 HCC admitted to King Chulalongkorn Memorial Hospital between January 2003 and December 2005 was carried out to select only patients who had unresectable HCC with vascular invasion and hepatic functions-Child-Pugh class-A. Vascular invasion was defined as involvement of portal vein, inferior vena cava (IVC), or their branches identified by imaging techniques. Non-surgical treatments were either transarterial chemoembolization (TACE) or systemic chemotherapy (SCT) in addition to general supportive care. Treatment outcomes of the patients were analyzed.

Results: Out of 71 unresectable HCC patients enrolled, 57patients were treated with TACE, while 14 received SCT. In the TACE group, 39 (68\%), 7 (12\%) and 11 (19\%) patients had portal vein, IVC, and both vessels invasion, respectively. In the SCT group; 11 (78\%), 1 (7\%) and 2 (14\%) had invasion of portal vein, IVC, and both vessels, respectively. Median overall survival in both groups was 158 days. Univariate analysis demonstrated that AFP level $<1000 \mathrm{ng} / \mathrm{mL}$, tumor size $<10 \mathrm{~cm}$, and SCT treatment significantly influenced survival. Additional multivariate analysis confirmed that diameters of tumor, and SCT were independent prognostic factors for good survival. A survival analysis showed longer survival in the SCT group than that of TACE (210 vs. 149 days, $\mathrm{p}=0.03$ ) group. Conclusion: Survival of HCC patients with major vessels invasion was better when treated with SCT compared to TACE. Future prospective study in larger populations to test the hypothesis is warranted.
\end{abstract}

Keywords: Hepatocellular carcinoma, systemic chemotherapy, transarterial chemoembolization

Hepatocellular carcinoma (HCC) is the most common cancer with the highest incidence in Asia and Africa [1]. Surgical resection is still the mainstay of treatment but fewer than $20 \%$ of patients are suitable for surgery [2]. Inadequate liver reserve postresection or advanced disease due to large tumor, multiple lesions, bilobar tumor, and vascular invasion are the main contraindications to surgery [3]. In general, major vascular invasion is considered a relative or absolute contraindication to liver resection. Transarterial chemoembolization (TACE) is the palliative treatment that demonstrates the survival

Correspondence to: Jade Suphapol, MD. Department of Surgery, Faculty of Medicine, Chulalongkorn University, Bangkok 10330, Thailand. E-mail: dr.jade@hotmail.com benefit in unresectable, especially large and multiple lesions HCC $[4,5]$. However, it is not suitable in advanced HCC with vascular invasion, especially main portal vein involvement or with systemic metastasis [6]. In patients with good liver function, systemic chemotherapy (SCT) is another option but recent studies did not demonstrate any survival benefit in advanced-HCC patients [2].

In this study, we analyzed outcome of nonsurgical treatment (TACE and SCT) in advanced-HCC patients with vascular invasion but with good liver functions in Thailand.

\section{Material and methods}

Between Jan 2003 and Dec 2005, 648 HCC patients were treated at the King Chulalongkorn 
Memorial Hospital. The diagnosis of HCC was based on persistently-raised serum alpha-fetoprotein level (>400 ng/mL) in conjunction with characteristic finding based on computed tomography (SCT), magnetic resonance imaging (MRI) or angiographic imaging. Patients who did not meet clinical criteria had fine-needle aspiration cytology to confirm diagnosis. During this period, 310 HCC patients were treated by TACE or systemic chemotherapy, and their medical records were reviewed to select only the patients who had unresectable HCC with vascular invasion at the time of treatment and hepatic functionsChild-Pugh class-A. Exclusion criteria included patients who had liver resection after downstaging of tumor by TACE, vascular invasion developing after courses of treatment, or liver function of Child-Pugh class B or C. Vascular invasion was defined as involvement of portal vein, inferior vena cava (IVC), or their branches identified by imaging techniques showing presence of tumor thrombus or occlusion of main or major branches of portal vein or IVC. TACE was offered as the palliative treatment in the patients except when total occlusions of main portal vein or significant artery-vein shunting were present. Patients with systemic metastasis or patients who were not suitable for TACE, or in whom TACE failed to arrest the progression of disease, were treated by SCT. Survival duration was counted from the start of treatment in each group.

All data of patients included age, sex, comorbid disease-diabetes mellitus, viral hepatitis profile, complete blood count, coagulogram, liver function tests, alpha-fetoprotein, and imaging studies before treatment. Imaging data included tumor size, number, lobar distribution, site of vascular invasion, evidence of cirrhosis and distant metastasis. Serious complication or adverse effects associated with the treatments were also recorded. Survival outcomes in all patients were recorded and analyzed.

We compared the survival outcome between patients treated using TACE and SCT.

\section{Statistical analysis}

Data analysis was performed using the SPSS program. Median survival was estimated using KaplanMeier method. Cox-regression model with proportional hazards was used to analyze factors associated survival outcome.

Chi square test or Student $t$ test was performed to compare baseline data between TACE and SCT groups. Difference of median survival between two groups was determined by the log-rank test, and survival plot graft was performed. P-value of less than 0.05 was considered statistically significant.

\section{Results}

Out of 310 HCC patients treated using TACE or SCT, 87 HCC patients had vascular invasion. Sixteen patients of this group were excluded due to failure of TACE, where no treatment in three cases, vascular invasion occurrence after TACE treatment in three cases, patient hepatic function Child-Pugh class B in three cases, and liver resection of tumor after downstaging by TACE in seven cases. Only 71 patients who had unresectable HCC with vascular invasion and with hepatic function Child Pugh class A were enrolled into this study. All patients had ECOG performance status $0-1$.

There were 54 (76.0\%) male patients and 17 (24.0\%) female patients. Mean age was 55 years (range: 26-78 years). Fifty-seven patients were treated with TACE, and 14 received CT. Ten patients (14.0\%) had diabetes. Nearly two-third of the patients had positive viral hepatitis-B marker, while eight patients (11.0\%) had positive viral hepatitis-C marker, and 17 patients (24.0\%) had none of B or C viral markers. Blood chemistries showed mean total bilirubin 1.16 $\mathrm{mg} / \mathrm{dL}$ (range: $0.9-2.4 \mathrm{mg} / \mathrm{dL}$ ), albumin $3.62 \mathrm{~g} / \mathrm{dL}$ (range: $2.8-4.8 \mathrm{~g} / \mathrm{dL}$ ), prothrombin time 13.3 seconds (range: 11.0-18.5 seconds), and platelet count 268457 cell/ $/ \mathrm{mm}^{3}$ (range: $60000-572000 \mathrm{cell} / \mathrm{mm}^{3}$. Mean alphafetoprotein was $52639 \mathrm{IU} / \mathrm{mL}$ (range: 1.62-500000 IU/ $\mathrm{mL}$ ). Imaging data showed unilobar tumor distribution in 53 patients $(74.6 \%)$ and bilobar in 18 patients (25.4\%).Tumor sizes ranged from 3 to $25 \mathrm{~cm}$ (mean: $11.5 \mathrm{~cm}$ ). Numbers of tumors were between one and seven nodules (mean: 1.6 nodules). Six patients (8.5\%) had previous history of rupture of tumor and 10 patients (14.5\%) had lung metastasis.

Portal involvement only was found in 50 patients (70.4\%). In this group, tumor was observed in the main portal vein in six patients. Only IVC involvement was demonstrated in eight patients (11.3\%). Involvement of both portal vein and outflow vessels (hepatic vein, IVC or both) was shown in 13 patients (18.3\%). IVC involvement was found in 16 patients (22.5\%). Median overall survival was 158 days.

Alpha-fetoprotein $<1000 \mathrm{IU} / \mathrm{mL}$, tumor size $<10 \mathrm{~cm}$, and SCT treatment influenced overall survival by univariate analysis as shown in Table 1. However, 
only tumor size and SCT treatment affected overall survival by the multivariate analysis as shown in Table 2.

Patients and tumor characteristics of both TACE and SCT groups were summarized in Table 3 . The patient characteristics were not different between two groups. Cirrhosis was found in 34 patients (59.6\%) of TACE and five patients (35.7\%) of SCT group. Mean tumor size and numbers of tumor in TACE and SCT group were 11.47, $11.63 \mathrm{~cm}$ and 1.6, 1.7, respectively. Portal vein, IVC and both vascular invasion were demonstrated in 39 (68.4\%), seven (12.3\%), and 11 (19.3\%) cases of TACE group and 11 (78.6\%), one (7.1\%), and two (14.3\%) cases in SCT group. The main portal vein invasion was found in three patients (5.3\%) patients in TACE group and three patients (21.4\%) in SCT group. Bilobar tumor involvement was observed significantly more frequently in SCT group (50\%) than in TACE group (19.3\%). Lung metastasis was detected in five patients in each group (8.8\% in TACE and $35.7 \%$ in SCT). In the five patients, TACE was employed because of marked abdominal discomfort. Most of them died within two months, thus, SCT had had not been offered. Four (7\%) patients in TACE group, and two $(14.3 \%)$ in SCT group had previous history of rupture tumor. Ten patients in TACE group had diabetes, and one patient in SCT group had hypertensive cardiomyopathy.

Table 1. Factor-associated survival outcome using the univariate analysis.

\begin{tabular}{|c|c|c|c|}
\hline \multirow{2}{*}{$\begin{array}{l}\text { Factors } \\
\text { Sex }\end{array}$} & \multicolumn{2}{|c|}{ Number of patients } & \multirow[t]{2}{*}{ P-value } \\
\hline & Male & 54 & \\
\hline & Female & 17 & NS \\
\hline \multirow[t]{3}{*}{ Viral hepatitis marker } & None & 17 & \\
\hline & $\mathrm{B}$ & 46 & NS \\
\hline & $\mathrm{C}$ & 8 & \\
\hline \multirow[t]{2}{*}{ Cirrhosis } & No & 32 & NS \\
\hline & Yes & 39 & \\
\hline \multirow[t]{2}{*}{ Alpha-fetoprotein (IU/mL) } & $<1000$ & 35 & 0.017 \\
\hline & $\geq 1000$ & 35 & \\
\hline \multirow[t]{2}{*}{ Tumor distribution } & Unilobar & 53 & NS \\
\hline & Bilobar & 18 & \\
\hline \multirow[t]{2}{*}{ Numbers of tumors } & $\mathrm{N}<3$ & 12 & NS \\
\hline & $\mathrm{N} \geq 3$ & 58 & \\
\hline \multirow[t]{2}{*}{ Size of tumor (cm) } & $\mathrm{T}<10$ & 27 & 0.015 \\
\hline & $\mathrm{T} \geq 10$ & 42 & \\
\hline \multicolumn{4}{|l|}{ Site of vascular involvement } \\
\hline \multirow[t]{2}{*}{ Portal vein } & Yes & 63 & NS \\
\hline & No & 18 & \\
\hline \multirow[t]{2}{*}{ Inferior vena cava (IVC) } & Yes & 16 & NS \\
\hline & No & 55 & \\
\hline \multirow[t]{2}{*}{ Treatment modality } & TACE & 57 & 0.033 \\
\hline & SCT & 14 & \\
\hline
\end{tabular}

NS=not significant.

Table 2. Factor-associated survival outcome using the multivariate analysis.

\begin{tabular}{lll}
\hline & P-value & Hazard ratio \\
\hline Tumor size (<10 vs. $\mathbf{1 0} \mathbf{~ c m )}$ & 0.020 & $2.028(1.11-3.70)$ \\
$\mathbf{S C T}$ & 0.021 & $0.390(0.18-0.86)$ \\
Alpha-fetoprotein & 0.084 & $1.647(0.94-2.90)$ \\
\hline
\end{tabular}


Table 3. Number of patients for various tumor characteristics in TACE and SCT. group.

\begin{tabular}{llll}
\hline & TACE (n=57) & SCT (n=14) & P-value \\
\hline Age (years) & 56 (range: $29-78)$ & 50 (range: $26-66)$ & 1.00 \\
Sex: Male & $43(75.4 \%)$ & $11(78.6 \%)$ & 0.62 \\
$\quad$ Female & $14(24.6 \%)$ & $3(32.4 \%)$ & 0.62 \\
HbsAg & $36(63.2 \%)$ & $10(71.4 \%)$ & \\
Anti-HCV & $6(10.5 \%)$ & $2(14.3 \%)$ & 0.18 \\
Cirrhosis & $34(59.6 \%)$ & $5(35.7 \%)$ & 0.70 \\
Alpha-fetoprotein & $55676($ range: & $40492($ range: $3.1-500000)$ & \\
(IU/mL) & $1.7-500000)$ & & 0.55 \\
TB(mg/dL) & $1.1($ range: $0.5-2.4)$ & $1.2($ range: $0.63-2.0)$ & 0.45 \\
Albumin (g/dL) & $3.6($ range: $2.8-4.8)$ & $3.7($ range: $3.1-4.2)$ & 0.52 \\
PT (second) & $13.3($ range: $11.3-15.7)$ & $13.0($ range: $11.2-15.8)$ & 0.56 \\
Platelet count (number) & $280410($ range: & $384603($ range: $96,000-$ & \\
Tumor size(cm) & $60,000-548,000)$ & $572,000)$ & 0.90 \\
Number of tumors & $11.4($ range: $3-25)$ & $11.6($ range: $8-15)$ & 0.74 \\
Unilobar & $1.6($ range: $1-7)$ & $1.7($ range: $1-3)$ & $0.03 *$ \\
Bilobar & $46(80.7 \%)$ & $7(50.0 \%)$ & 0.33 \\
Rupture & $11(19.3 \%)$ & $7(50.0 \%)$ & 0.21 \\
Lung metastasis & $4(7 \%)$ & $2(14.3 \%)$ & 0.09 \\
Vascular invasion & $5(8.8 \%)$ & $5(35.7 \%)$ & 0.16 \\
$\quad$ Portal vein & & & \\
$\quad$ Inferior vena cava & $39(64.4 \%)$ & $11(78.6 \%)$ & \\
$\quad$ Both & $7(12.3 \%)$ & $2(14.3 \%)$ & \\
Main portal vein & $11(19.3 \%)$ & $1(7.1 \%)$ & \\
Inferior vena cava & $3(5.3 \%)$ & & \\
\hline
\end{tabular}

HbsAg=hepatitis B surface antigen, TB=total bilirubin, PT=prothrombin time, *significant.

\section{Treatment}

TACE was done by injection of $5 \mathrm{~mL}$ of an emulsion of lipiodol, $20 \mathrm{mg}$ of mitomycin $\mathrm{C}$ and 500mgof 5-FU into feeding artery and followed by gelfoam embolization. TACE was performed every four to six weeks. CT (computed tomography) scanning was done to evaluate response three to four weeks after every sessions of TACE.

In SCT group, different regimens were employed. Nine cases received doxorubicin regimen that consisted of IV administration of doxorubicin maximum $60 \mathrm{mg} / \mathrm{m}^{2}$ on day one every four weeks. One patient received doxorubicin plus gemcitabine. Two patients received gemcitabine $1000 \mathrm{mg} / \mathrm{m}^{2}$ on day 1 plus oxaliplatin $100 \mathrm{mg} / \mathrm{m}^{2}$ on day 2 every two weeks, and after completion, doxorubicin regimen was added in one patient. One patient received six courses of doxorubicin regimen followed by cisplatinum 60 $\mathrm{mg} / \mathrm{m}^{2}$ on day 1 every four weeks. Treatment was repeated until disease progression, unacceptable toxicity was detected, or up to maximum six cycles. Only one patient received capecitabine $1000 \mathrm{mg} / \mathrm{m}^{2}$ orally twice daily of two weeks followed by a oneweek rest for 13 cycles. Dosage of the subsequent cycles was adjusted according to prior liver function test and the toxic effects that developed during the preceding cycle. All patients received standard supportive regimen consisting of hydration and antiemetics.

Patients in TACE group received one to eight treatment sessions (median 2). In SCT group, nine patients had at least a session of TACE before treatment (range: 1-4 sessions, median 1).

Liver function deterioration occurred in two patients of TACE group. Both of them had main portal vein invasion and died within a month. Postembolization syndrome in one patient required admission after a session of TACE. In SCT group, only one patient developed febrile neutropenia after one course of doxorubicin. 


\section{Survival}

At the end of study, four patients in CT group and eight patients in TACE group were still alive. Median survival in TACE group was 149 days, while median survival in CT group was 210 days $(\mathrm{p}=0.03)$.

\section{Discussion}

Hepatocellular carcinoma (HCC) tends to invade the intrahepatic vasculatures such as portal vein, hepatic vein, or IVC [7-9]. The rate of portal vein invasion is $34 \%$ to $40 \%$ [10]. The median survival of HCC patients with portal vein tumor thrombus was reported to be 2.7 months [11].

The management of HCC with vascular involvement is complicated and controversial. Recently, aggressive surgical management has been reported frequently [1, 3, 10, 12-14]. Minagawa et al. [10] reported favorable results from hepatectomy in HCC patients with portal vein tumor thrombus. In their report, mean duration of survival was $3.4 \pm 2.7$ years in 18 patients who received transcatheter arterial embolization and hepatectomy, and $0.36 \pm 0.26$ years in 27 patients who did not receive hepatectomy. Their criteria used for hepatectomy were tumors with less than two nodules, and portal trunk not occluded by tumor thrombus and ICG-R rate 15 minutes $<20 \%$. Ohkubo et al. [12] recommended curative liver resection in HCC patients with macroscopic portal vein tumor thrombosis for tumor size $<10 \mathrm{~cm}$, absence of intrahepatic metastasis. However, unresectable HCC patients with vascular invasion have still poor outcome.

According to Barcelona-Clinic Liver Cancer Staging System [15], HCC with vascular invasion are classified as "advanced (stage C)", where treatment is recommended only in controlled trial settings. However, palliative treatment, either TACE or SCT, may be required in a sub-group of the patients with good liver function.

The present study focused on unresectable HCC with vascular invasion at the time of treatment and patient hepatic functions-Child-Pugh class A. Our obtained results demonstrated median overall survival of 158 days (95\% confidential interval: 114-201 days). This was not different from other reports [16-18]. Using the univariate analysis, gender, viral hepatitis markers, cirrhosis, tumor distribution (unilobar/bilobar), number of tumor $(<3 / \geq 3)$, site of vascular invasion (portal vein/IVC) did not affect the survival outcomes.
Only alphafetoprotein level ( $<1000 \mathrm{IU} / \mathrm{mL})$, tumor size $<10 \mathrm{~cm}$, and treatment modality (SCT group) were good prognostic factors. However, our multivariate analysis showed that tumor size $\geq 10 \mathrm{~cm}$ was the negative prognostic factor and treatment modality by SCT was a good prognostic factor.

Pawlik et al. [19] demonstrated that size of tumor was associated with the occurence of vascular invasion, that is, the larger the tumor $(\geq 5 \mathrm{~cm} /<5 \mathrm{~cm})$, the greater occurence of vascular invasion (55\% vs. 31\%). This could explain why tumor size $\geq 10 \mathrm{~cm}$ in our analysis was the negative prognostic factor. Median survival in SCT group was 210 days compared with 149 days in TACE group, which was statistically significant $(p=0.03)$. This could be due to the detrimental effect of TACE on HCC patients with vascular invasion. Bruix et al. [4] stated that HCC patients with thrombosis of a lobar or segmental branch constituted poor candidates for TACE as TACE in these patients could occlude the blood supply of non-tumoral liver causing ischemia, leading to liver failure. However, there were only two patients in our TACE group who developed liver failure after TACE and died within 30 days.

Role of STC in HCC patients is limited. Median survival of HCC patients with portal vein involvement who received chemotherapy (systematic or regional) was reported to be 5.6 [16] and 9.2 months [20], respectively. There is no evidence to suggest that STC improves overall survival [2]. Response rate of STC in previous studies was $10-20 \%$ depending on the regimen use [18, 21, 22]. Kaneko et al. [23] and Ishikawa et al. [24] showed survival benefit in HCC with portal vein thrombosis only compared to supportive care alone. Tzoracoleftherakis et al. [17] reported a slight but not statistically significant superiority of intra-arteial chemotherapy against SCT for non-resectable HCC (stage IV A). Our data supported the role of STC rather than TACE in HCC patients who had vascular invasion but good liver function.

In conclusion, unresectable HCC patients with major vascular invasion had grave prognosis. However, HCC patients with major vascular invasion and ChildPugh class-A who had tumor size $<10 \mathrm{~cm}$ may benefit from palliative treatment by STC. Prospective randomized controlled trials are needed to support this strategy. 


\section{Reference}

1. Fan J, Zhou J, Wu Z-Q, Qiu S-J, Wang X-Y, Shi Y-H, et al. Efficacy of different treatment strategies for hepatocellular carcinoma with portal vein tumor thrombosis. World J Gastroenterol. 2005; 11:1215-9.

2. Nowak AK, Chow PK, Findlay M. Systemic therapy for advanced hepatocellular carcinoma: a review. Eur J Cancer. 2004; 40:1474-84.

3. Gotohda N, Kinoshita T, Konishi M, Nakagohri T, Takahashi S, Furuse J, et al. New indication for reduction surgery in patients with advanced hepatocellular carcinoma with major vascular involvement. World J Surg. 2006; 30:431-8.

4. Bruix J, Sala M, Llovet JM, Chemoembolization for hepatocellular carcinoma. Gastroenterology. 2004; 127:179-88.

5. Llovet JM, Bruix J. Systematic review of randomized trials for unresectable hepatocellular carcinoma: chemoembolization improved survival. Hepatology. 2003; 37:429-42.

6. O’Suilleabhain CB, Poon RT, Yong JL, Ooi GC, Tso WK, Fan ST. Factors predictive of 5-year survival after transarterial chemoembolization for inoperable hepatocellular carcinoma. Br J Surg. 2003; 90:325-31.

7. Okuda K, Ohtsuki T, Obata H, Tomimatsu M, Okazaki $\mathrm{N}$, Hasegawa H, et al. Natural history of hepatocellular carcinoma and prognosis in relation to treatment. Study of 850 patients. Cancer. 1985; 56:918-28.

8. The Cancer of the Liver Italian Program (CLIP) Investigators. Prospective validation of the CLIP score: a new prognostic system for patients with cirrhosis and hepatocellular carcinoma. Hepatology. 2000; 31: 840-5.

9. Greten TF, Papendorf F, Bleck JS, Kirchhoff T, Wohlberedt T, Kubicka S, et al. Survival rate in patients with hepatocellular carcinoma: a retrospective analysis of 389 patients. Br J Cancer. 2005; 92:1862-8.

10. Minagawa M, Makuuchi M, Takayama T, Ohtomo K. Selection criteria for hepatectomy in patients with hepatocellular carcinoma and portal vein tumor thrombus. Ann Surg. 2001; 233:379-84.

11. Llovet JM, Bustamante J, Castells A, Vilana R, Ayuso Mdel C, Sala M, et al. Natural History of untreated nonsurgical hepatocellular carcinoma: rationale for the design and evaluation of therapeutic trials. Hepatology. 1999; 29:62-7.

12. Ohkubo T, Yamamoto J, Sugawara Y, Shimada K, Yamasaki S, Makuuchi M, et al. Surgical results for hepatocellular carcinoma with macroscopic portal vein tumor thrombosis. J Am Coll Surg. 2000; 191:657-60.

13. Pawlik TM, Poon RT, Abdalla EK, Ikai I, Nagorney
DM, Belghiti J, et al. Hepatectomy for hepatocellular carcinoma with major portal or hepatic vein invasion: results of a multicenter study. Surgery. 2005; 137: 403-10.

14. Ikai I, Hatano E, Hasegawa S, Fujii H, Taura K, Uyama $\mathrm{N}$, et al. Prognostic index for patients with hepatocellular carcinoma combined with tumor thrombosis in the main portal vein. J Am Coll Surg. 2006; 202:431-8.

15. Llovet JM, Burroughs A, Bruix J. Hepatocellular carcinoma. Lancet. 2003; 362:1907-17.

16. Okada S, Okazaki N, Nose H, Yoshimori M, Aoki K. Prognostic factors in patients with hepatocellular carcinoma receiving systemic chemotherapy. Hepatology. 1992; 16:112-7.

17. Tzoracoleftherakis EE, Spiliotis JD, Kyriakopoulou T, Kakkos SK. Intra-arterial versus systemic chemotherapy for non-operable hepatocellular carcinoma. Hepatogastroenterol. 1999; 46:1122-5.

18. Yang T-S, Chang H-K, Chen J-S, Lin Y-C, Liau C-T, Chang W-C. Chemotherapy using 5-fluorouracil, mitoxantrone and cisplatin for patients with advanced hepatocellular carcinoma: an analysis of 63 cases. J Gastroenterol. 2004; 39:362-9.

19. Pawlik TM, Delman KA, Vauthey J-N, Nagorney DM, Ng IO-L, Ikai I, et al. Tumor size predicts vascular invasion and histologic grade: implications for selection of surgical treatment for hepatocellular carcinoma. Liver Transpl. 2005; 11:1086-92.

20. Ando E, Yamashita F, Tanaka M, Tanikawa K. A novel chemotherapy for advanced hepatocellular carcinoma with tumor thrombosis of the main trunk of the portal vein. Cancer.1997; 79:1890-6.

21. Taieb J, Bonyhay L, Golli L, Ducreux M, Boleslawski E, Tigaud JM, et al. Gemcitabine plus oxaliplatin for patients with advanced hepatocellular carcinoma using two different schedules. Cancer. 2003; 98:2664-70.

22. Lozano RD, Patt YZ, Hassan MM. Oral capectabine (Xeroda) for the treatment of hepatobiliary cancers (hepatocellular carcinoma, cholangiocarcinoma, and gallbladder cancer). Proc Annu Meet Am Soc Clin Oncol. 2000; 22:A1407.

23. Kaneko S, Urabe T, Kobayashi K. Combination chemotherapy for advanced hepatocellular carcinoma complicated by major portal vein thrombosis. Oncology. 2002; 62 (Suppl 1): 69-73.

24. Ishikawa T, Ichida T, Sugitani S, Tsuboi Y, Genda T, Sugahara S, et al. Improved survival with oral administration of enteric-coated tegafur/uracil for advanced stage IV-A hepatocellular carcinoma. J Gastroenterol Hepatol. 2001; 16:452-9. 